\section{Values-based action in fibromyalgia: results from a randomized pilot of acceptance and commitment therapy}

\author{
Jennifer L. Steiner, 1 Leah Bogusch,2 \\ Silvia M. Bigatti ${ }^{3}$
}

1Atlanta Veteran's Affarirs Medical

Center, GA; 2Department of Psychology, Indiana University-Purdue University

Indianapolis, IN; 3Indiana University

School of Public Health, Indianapolis, IN, USA

\section{Abstract}

Fibromyalgia Syndrome (FMS) is a chronic pain condition characterized by pain, fatigue, and nonrestorative sleep. The disruptive symptoms of FMS are associated with reductions in quality of life related to family, intimate relationships, and work. The present study was part of a randomized pilot study of an 8-week Acceptance and Commitment Therapy (ACT) intervention compared to education in a sample of 28 women with FMS. The Chronic Pain Values Inventory was administered at baseline, postintervention, and 12 week follow-up. Both groups showed significant improvements in family success, which were maintained at follow-up. Groups showed a differential pattern of success in work. The ACT group demonstrated significant, maintained improvements in success in intimate relationships, while the education group reported no changes over time. Findings suggest that both interventions may lead to improvements in valued living; however different interventions may be best suited for certain valued domains. The results of this study indicate that FMS patients are able to improve their success in family and intimate relationships and losses in these areas are not necessarily permanent.

\section{Introduction}

Fibromyalgia Syndrome (FMS) is a chronic pain condition characterized by recurrent musculoskeletal pain, fatigue, and nonrestorative sleep. FMS is estimated to affect approximately $2-7 \%$ of the general population and is most commonly diagnosed in women over the age of $40 .{ }^{1}$ A common symptom is hypersensitivity to touch or tactile stimuli, ${ }^{2}$ which can make daily actions like shaking hands or hugging aversive; thus deterring these interpersonal actions. Hypersentivity also impacts exe- cuting physical tasks such as household cleaning. ${ }^{3}$ Because chronic, unrelenting pain is the primary symptom of FMS, many FMS patients experience losses in physical and social functioning, ${ }^{3}$ and overall quality of life domains.

\section{Impact of fibromyalgia syndrome on multiple life domains}

FMS often has a negative impact on patient relationships with family members, which in turn may exacerbate symptoms. Preece and Sandberg found increases in family strain were associated with increased symptom impact. Romano, Turner, and Jenson demonstrated that chronic pain patients and their spouses reported significantly lower levels of family cohesion compared to healthy couples. 4,5 FMS patients often have difficulty carrying out previously held responsibilities to the family, such as completing housework or transporting children to activities; 6 thus, FMS forces a lifestyle modification for the entire family.

In addition to impacting the family unit, FMS has been associated with strained relationships, increases in stress, and emotional turmoil for both partners in intimate relationships. ${ }^{7}$ Resentment may result from perceptions of unfair divisions of labor, ${ }^{8}$ such as spouses covering household tasks and social responsibilities that their partners can no longer carry out.9-11 FMS has also been associated with lower marital satisfaction in patients' spouses. ${ }^{8}$ Decreased marital satisfaction may be related to decreases in sexual functioning which are common among women with FMS. 12 It is clear, that FMS can have a profound impact on various aspects of an individual's romantic relationship.

FMS often prevents patients from maintaining regular employment or performing at work without painful results. Research suggests unemployment rates increase following a FMS diagnosis. ${ }^{13}$ FMS patients are employed at a lower rate than the overall population 12 and report high rates of disability awards. 14

\section{Approaches to the problem}

While many treatment options have focused on either symptom reduction or management, these attempts have not always translated into success in quality of life domains. Acceptance and Commitment Therapy [Acceptance and Commitment Therapy (ACT) ] $]^{15,16}$ is a promising intervention to address this problem. ACT is a psychotherapy aimed at decreasing unsuccessful and maladaptive attempts to avoid or control pain and suffering. ${ }^{15,16}$ ACT focuses on encouraging engagement in behaviors that are in service of working toward one's life values, thus enhancing quality of life. ACT is different from other cognitive-based therapy models in that ACT teaches patients to accept pain without attempts to control the pain, as these
Correspondence: Silvia M. Bigatti, Indiana University Richard M. Fairbanks School of Public Health at IUPUI, 714 N Senate Avenue, EF250, IN, USA.

Tel. +1.317.274.6754 - Fax: +1.317.274.3443

E-mail: sbigatti@iupui.edu

Key words: fibromyalgia, acceptance and commitment therapy, quality of life.

Acknowledgments: the authors would like to acknowledge the staff of the IU Clinical Research Center for Pain-Fibromyalgia for their assistance in recruitment and their commitment to fibromyalgia research.

Contributions: JLS, conception, design, implementation of this study and the parent pilot study, data analysis and portions of text writing, drafting and revision process; LB, conception of the hypotheses for the present work, data analysis, portions of text writing and drafting process; SMB conception and design of this study and the parent pilot study, drafting and revision process, portions of the text writing.

Conflict of interests: the authors declare no potential conflict of interests.

Conference presentation: this data was originally presented at Indiana University-Purdue University, Indianapolis' 2012 Annual Research Day Conference. This data represents a sub study of a pilot study designed to assess the feasibility and acceptability of Acceptance and Commitment Therapy for women with Fibromyalgia.

Received for publication: 30 March 2013.

Revision received: 22 May 2013.

Accepted for publication: 2 June 2013.

This work is licensed under a Creative Commons Attribution NonCommercial 3.0 License (CC BYNC 3.0).

(C) Copyright J.L. Steiner et al., 2013

Licensee PAGEPress, Italy

Health Psychology Research 2013; 1:e34

doi:10.4082/hpr.2013.e34

behaviors often interfere with other important activities (i.e. choosing rest over work or time with family). This philosophy is supported by research demonstrating that increased acceptance of pain is associated with increased wellbeing and decreased pain severity, ${ }^{17}$ as well as improvements in work status. ${ }^{18,19}$

Although ACT and values based action has been studied in mixed chronic pain samples, it has yet to be explored in FMS. The present study aimed to explore the effects of ACT on success in valued-based action in a FMS sample. We hypothesized that ACT would produce changes in success across life domains, and that these improvements would be maintained at 12-week follow-up (Figure 1). 


\section{Materials and Methods}

\section{Recruitment}

Participants were 28 women with a diagnosis of FMS. Recruitment efforts included flyers, doctor referrals, referrals from the IU Clinical Research Center for Fibromyalgia-Pain, newspaper advertisements, and a general Rheumatology recruitment database. Potential participants from the recruitment database or physician/center referral were contacted via telephone call and asked to participate in the study. Volunteers who received a flyer contacted the study manager via telephone or email.

During the initial contact, the study manager explained the study purpose and the commitment required. If interested in participation, the individual was completed a brief 10 15 minute questionnaire to determine eligibility for the study. This was completed either on the telephone, or online using a secure data capture system called REDCAP. Inclusion criteria were as follows: i) a diagnosis of FMS by a physician, ii) a score at 40 or above on the FIQ to allow for intervention effects, iii) age between 18-65, iv) ability to attend 1-hour weekly sessions for 8 weeks and to engage in home practice, v) willingness to be randomized into two different intervention groups, vi) on stable doses of the following medications for at least 4 weeks: cyclobenzaprine, tramadol, gabapentin, pregabalin, tricyclics, selective serotonin reuptake inhibitor, and selective norepinephrine serotonin reuptake inhibitor to prevent potential confounding of medication on outcomes, and vii) ability to speak and write in English. Exclusion criteria included: i) active suicidal intention or plan, ii) other major rheumatic conditions, iii) schizophrenia or other psychosis (self-report and clinician assessment), and iv) dementia neuropsychological problems, or cognitive impairment (self-report and clinician assessment). Those individuals deemed eligible continued on to complete an online informed consent statement and participate in the rest of the study. A second informed consent was signed during the initial therapy session. We were able to make direct contact with 77 women, of which 56 agreed to be assessed for eligibility. Women refused participation for several reasons: 10 reported they were doing well/not interested in other treatment options, 8 due to time commitment, and 3 did not have travel accommodations. Of the 56 who agreed, 37 met criteria; 9 decided not to participate after the screening and scheduling process. This resulted in a total sample size of 28 women and a recruitment rate of $61 \%$.

\section{Procedure}

The present study is a secondary data analy- sis with data collected from a pilot study of Acceptance and Commitment Therapy for FMS. This small scale randomized controlled trial (RCT) used the same sample as that in the present analyses. Participants were randomly assigned to receive ACT or FMS/Pain management Education. Once randomized, participants completed approximately 8 one-on-one sessions with either the ACT interventionist or the FMS educator. Sessions occurred once a week for 1 hour at a time. The primary purpose of the parent study was to assess the feasibility and acceptability of an ACT intervention for FMS. In addition to completing the measure that is the focus of the present work, participants completed a battery of self report measures related to physical functioning and various psychosocial variables.

The manualized ACT intervention was based on the ACT manual, Living Beyond Your Pain: Using Acceptance and Commitment Therapy to Ease Chronic Pain. ${ }^{20}$ The intervention included the following components: (a) assessment, conceptualization, rationale, and rapport; (b) willingness to contact unwanted emotion (c) introduction to cognitive defusion (d) introduction to values and values clarification; introduction to committed action (e) willingness, mindfulness, present moment, and defusion (f) further establishing values (g) committed action; (h) self as context and committed action. According to ACT experts, ACT components are simultaneously present in varying degrees in all sessions and are largely dependent on one another; thus, ACT is often less sequential than other interventions. Flexibility in presentation order was built into the protocol. However, a suggested order of sessions was encouraged as a framework for the intervention.

The education intervention consisted of the following topics: a) overview of FMS, b) pain, c) fatigue, d) sleep, e) stress, f), nutrition, e) living well with FMS. This manualized intervention was adapted from a manual ${ }^{9}$ that has successfully been used in other RCTs with FMS patients. ${ }^{21}$ Participants in the education intervention were provided with a written copy of the educational material at each session; the participant and interventionist discussed the material together. Information was provided in a conversational manner; the interventionist asked standardized questions to elicit the participants' reactions to the material. Interventionists encouraged participants to relate the material to their own experience.

All sessions of both interventions were audio recorded and audited for fidelity to the intervention. Audits ensured that interventionists were adhering to the manual and that there were no cross-contamination effects from one intervention to another. Additionally, interventionists were not trained in the opposing intervention in order to guard against possible contamination. Finally, ACT interventionists received weekly supervision from an ACT expert to assist in maintaining the integrity of the intervention.

Participants completed a self-report battery at three time points: immediately before the first session, directly following the last session, and twelve weeks after the last session.

\section{Measures \\ Chronic Pain Values Inventory}

The [Chronic Pain Values Inventory (CPVI) $]^{22}$ measures the effects of chronic pain on a person's values. The measure consists of six, single item domains. The six domains are Family, Intimate Relations, Friends, Health, Work, and Personal Growth and Learning. Importance is rated on scale of 0-5 (0 = Not at all important; 5 = Extremely important). Participants are also asked to rate success in each domain, using a 0-5 scale $(0=$ Not at all

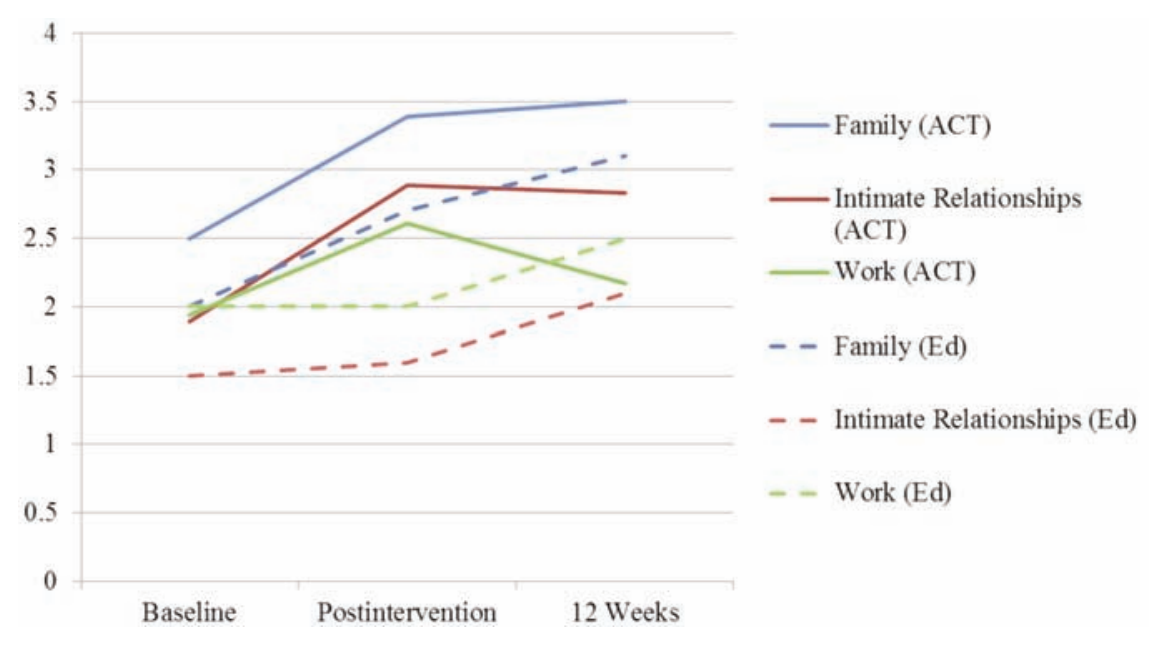

Figure 1. Change in success overtime. 
successful; 5 = Extremely successful). The CPVI has shown good reliability, with $\alpha=0.82$ for both importance and success subscales. The CVPI has demonstrated discriminant validity when compared to a measure of experiential avoidance, the Pain Anxiety Symptoms Scale, $r=-0.18, \mathrm{P}<0.05$, and concurrent with the Chronic Pain Acceptance Questionnaire, a measure of acceptance, $r=0.49, \mathrm{P}<0.001 .23$ These relationships make sense from a theoretical perspective given that avoidance behaviors are typically antithetical to valued based living; conversely, valued based action is believed to be facilitated by acceptance of suffering. 16

\section{Statistical analyses}

The data was checked for outliers and missing values prior to analysis. Data was analyzed as an intent-to-treat using the last measure carried forward method. ANCOVA was used to assess group differences in success at postintervention and at 12-week follow-up; baseline success ratings were entered as the covariate in order to control for this value. Paired sample t-tests were used to examine significant differences within groups over time. Of note, while it is generally advised to conduct an adjustment (such as a Bonferroni adjustment) to account for multiple comparisons when many tests are run, we opted against this adjustment. The Bonferroni adjustment is capable of reducing type I error through deflating the overall $\alpha$ applied to each test that is run. Statistical adjustments reduce type I error; however, the chance of falsely failing to reject the null hypothesis and failure to detect an effect that actually exists is increased. In cases of very small sample size where detection of group differences is already challenging because of lack of power (such as in the present study), an adjustment for type I error could potentially mask existing effects. ${ }^{24}$ Recent recommendations have suggested providing effect sizes to aid in determining the relevance and clinical significance of a given effect. ${ }^{24}$ Thus, it was decided to not include an adjustment for type I error, and effect sizes are provided in accordance with recommendations.

\section{Results}

\section{Demographics}

Of the 28 participants, 18 were randomized to ACT and 15 to education. Between the time of randomization and baseline assessment 5 participants dropped out of the education group. There were no significant differences between groups on pain intensity or any demographic variables at baseline (Table 1). Average current pain intensity for the entire sample was 6.64 ( $\mathrm{SD}=2.02)$. The sample was predominantly Caucasian (79.3\%). The mean age of participants was 48.63 years ( $\mathrm{SD}=12.96) ; 39.4 \%$ were on some form of disability, $14.3 \%$ had reduced work hours, and $21.4 \%$ had stopped working due to FMS but had not received disability. In total, $75.1 \%$ of the sample experienced disrupted work. At baseline, there were no significant differences between the ACT and education group (Table 2) in any variables of interest. Of note, the analyses were conducted both as mentioned above and with interventionist as a covariate to account for possible changes due to interventionist factors rather than the intervention itself. The pattern of results did not change when interventionist was included as a covariate, thus the analyses presented are without this covariate for the purposes of fostering greater statistical power.

\section{Family}

Analyses revealed no significant group differences at post-intervention nor at the 12

Table 1. Demographic characteristics of participants by treatment group.

\begin{tabular}{|c|c|c|c|}
\hline Variable & $\begin{array}{c}\text { ACT }(\mathrm{n}=18) \\
\text { Means (SD)/frequencies, \% }\end{array}$ & $\begin{array}{c}\text { Education }(\mathrm{n}=10) \\
\text { Means (SD)/frequencies, \% }\end{array}$ & Group comparisons \\
\hline Age of participants & $47.82(12.91)$ & $50.00(13.62)$ & $\mathrm{t}(26)=0.415, \mathrm{P}=0.682$ \\
\hline Current pain & $6.29(2.32)$ & $7.30(1.16)$ & $\mathrm{t}(26)=1.30, \mathrm{P}=0.206$ \\
\hline Average pain & $7.00(2.40)$ & $7.60(1.90)$ & $\mathrm{t}(26)=0.679, \mathrm{P}=0.503$ \\
\hline $\begin{array}{l}\text { Race/ethnicity } \\
\text { White non-hispanic } \\
\text { Non-white }\end{array}$ & $\begin{array}{l}77.8 \\
22.2\end{array}$ & $\begin{array}{l}80.0 \\
20.0\end{array}$ & $\begin{array}{c}x^{2}=(1,27)=0.45 \\
P=0.831\end{array}$ \\
\hline $\begin{array}{l}\text { Employment status } \\
\text { Employed } \\
\text { Not employed }\end{array}$ & $\begin{array}{l}38.9 \\
61.1\end{array}$ & $\begin{array}{l}40.0 \\
60.0\end{array}$ & $\begin{array}{c}x^{2}=(1,27)=0.004 \\
\mathrm{P}=0.952\end{array}$ \\
\hline $\begin{array}{l}\text { Marital status } \\
\text { Married/partnered } \\
\text { Not partnered }\end{array}$ & $\begin{array}{l}61.1 \\
38.9\end{array}$ & $\begin{array}{l}40.0 \\
60.0\end{array}$ & $\begin{array}{c}x^{2}=(1,27)=0.894 \\
P=0.345\end{array}$ \\
\hline
\end{tabular}

Table 2. Changes in success in valued life domains over time by group.

\begin{tabular}{|c|c|c|c|c|c|c|c|c|c|}
\hline & $\begin{array}{l}\text { Baseline M } \\
\text { (SD) }\end{array}$ & $\begin{array}{l}\text { Post M } \\
\text { (SD) }\end{array}$ & $\begin{array}{l}12 \text { week } \\
\text { follow-up }\end{array}$ & $\begin{array}{l}\text { Baseline-post } \\
\text { paired t-test }\end{array}$ & $\mathbf{P}$ & d & $\begin{array}{c}\text { Baseline-week t-test } \\
12 \text { paired }\end{array}$ & $\mathbf{P}$ & d \\
\hline \multicolumn{10}{|c|}{ ACT } \\
\hline Family & $2.50(1.50)$ & $3.39(1.24)$ & $3.50(1.47)$ & $\mathrm{t}(17)=3.19$ & $0.005^{*}$ & 0.75 & $\mathrm{t}(17)=3.43$ & $0.003^{*}$ & 0.81 \\
\hline Intimate relationships & $1.89(1.32)$ & $2.89(1.41)$ & $2.83(1.42)$ & $t(17)=2.70$ & $0.015^{* *}$ & 0.64 & $t(17)=2.27$ & $0.036^{* *}$ & 0.53 \\
\hline Work & $1.94(1.35)$ & $2.61(1.54)$ & $2.17(1.50)$ & $t(17)=2.75$ & $0.014^{* *}$ & 0.64 & $t(17)=0.64$ & 0.532 & 0.15 \\
\hline \multicolumn{10}{|c|}{ Education } \\
\hline Family & $2.00(1.25)$ & $2.70(1.57)$ & $3.10(1.66)$ & $\mathrm{t}(9)=1.91$ & 0.089 & 0.60 & $\mathrm{t}(9)=2.18$ & 0.057 & 0.69 \\
\hline Intimate relationships & $1.50(1.43)$ & $1.60(1.17)$ & $2.10(1.60)$ & $\mathrm{t}(9)=0.26$ & 0.798 & 0.08 & $\mathrm{t}(9)=0.97$ & 0.357 & 0.31 \\
\hline Work & $2.00(1.25)$ & $2.00(0.94)$ & $2.50(1.35)$ & $\mathrm{t}(9)=0.00$ & 1.000 & 0.00 & $\mathrm{t}(9)=0.86$ & 0.413 & 0.27 \\
\hline
\end{tabular}

*Significant at $\alpha<0.01$; **Significant at $\alpha<0.05$. 
week follow-up (Table 3). Paired t-tests revealed that both the ACT group and the education group demonstrated the same pattern of change over time. This pattern shows a significant within group difference from baseline to postintervention (Table 2), and a significant within group difference from baseline to 12 week follow-up. However, no significant difference was detected from post-intervention to 12 week follow-up; thus changes obtained by post intervention were maintained at the 12 week follow-up.

\section{Intimate relations}

Analyses revealed a significant between groups difference from baseline to post-intervention (Table 3). However, at the 12 week follow-up no statistically significant differences were observed. Paired t-tests revealed that the ACT group reported significant increases in success with intimate relationships from baseline to post intervention (Table 2), and from baseline to 12 week follow-up. However, there was no significant change between postintervention and follow-up, indicating maintained improvements. In contrast, the education group did not demonstrate any significant change over time (Table 2).

\section{Work}

Analyses revealed no significant group differences at post-intervention nor at the 12 week follow-up (Table 3). Paired t-tests revealed that the ACT group and the education group demonstrated different patterns of change over time. For the ACT group, there was a significant increase in success at work from baseline to postintervention (Table 2). However, there was no significant change from baseline to follow-up, indicating that improvements regressed back toward baseline. For the education group, no significant changes were observed over time (Table 2).

\section{Discussion}

This study examined the changes in success with family, intimate relationships, and work in a sample of women with FMS who received either ACT or education about symptom selfmanagement. We hypothesized that following an 8-week intervention period those who received ACT would report statistically greater increases in success compared to the education group. Furthermore, we hypothesized that these increases would be maintained 12 weeks following the intervention. Our hypothesis was supported for the domain of intimate relationships. The ACT group increased their average success by a full point on the CVPI, from not at all successful to approaching moderately successful. The education group reported success that was unchanged at post-intervention. These findings are encouraging given that improvements in that the ACT group were maintained. Previous research on ACT has demonstrated it to be effective in reducing interpersonal distress within couples and helping individuals to act in ways that are consistent with their relationship values. ${ }^{24}$ There has been no research in this area specific to a medical or chronic pain population. Given that intimate relationships are an area that is typically negatively impacted by FMS, 7,12 ACT may prove to be a promising intervention to help women with FMS improve their own behavior in their relationship.

Success in family and work domains followed a different pattern than expected. Contrary to our hypothesis, there were no significant group differences in either the family or work domain. Regarding family, both groups showed increases in success at postintervention and continued improvement at 12 week follow-up. For both groups, this reflected a change in reported success from somewhat successful to moderately successful over the course of the study. These results are congru-

Table 3. Results of ANCOVA analyses.

\begin{tabular}{|c|c|c|c|c|}
\hline & $\mathrm{F}$ & Df & $P$ & Partial $\eta 2$ \\
\hline \multicolumn{5}{|c|}{ Postintervention } \\
\hline Family & 0.794 & $(1,28)$ & 0.381 & 0.031 \\
\hline Intimate relationships & 5.279 & $(1,28)$ & $0.030^{*}$ & 0.174 \\
\hline Work & 3.176 & $(1,28)$ & 0.087 & 0.113 \\
\hline \multicolumn{5}{|c|}{ t12 week follow-up } \\
\hline Family & 0.031 & $(1,28)$ & 0.862 & 0.001 \\
\hline Intimate relationships & 1.231 & $(1,28)$ & 0.278 & 0.047 \\
\hline Work & 0.321 & $(1,28)$ & 0.576 & 0.013 \\
\hline
\end{tabular}

ent with values-based action in other chronic pain samples following an acceptance protocol.25 Of note, neither intervention was more effective than the other in producing increased success in family life. In fact, both groups demonstrated improvements in this domain, regardless of intervention. Thus, it may be that both ACT and education are viable candidates for targeting improvement in family life; yet, it is possible that the interventions work through different mechanisms. ACT may increase success through emphasizing values consistent behavior, thus participants may have engaged in family oriented behavior as a result of identifying this as a life value. Education may have taught more effective strategies for symptoms management than previously known, creating more time with family. This assumption is purely speculative; however, qualitative data analysis may shed some light on this issue. Research suggests that education in self-management strategies can produce similar effects as behavioral interventions, ${ }^{26}$ motivational interventions, ${ }^{21}$ and a variety of other nonpharmacologic interventions, ${ }^{27}$ thus education may be a more cost effective alternative to ACT.

Given that the groups did not differ in family relations but did in intimate relationships, this may suggest a more specialized intervention such as ACT may be indicated for more complex domains. This may especially true for romantic relationships; previous research suggests that although education or interdisciplinary treatments may improve many areas of functioning in FMS patients, martial satisfaction is not effected. 28 However, ACT has demonstrated preliminary effectiveness in case studies of couples in relationship distress, ${ }^{24}$ these findings are consistent those presented. In addition to possibly being better suited for improvement in romantic relationships, ACT may have some practical advantages to education as well. Other studies with chronic pain patient that have compared ACT to evidence-based interventions (such as CBT) have found equivalent benefit; however those in the ACT group reported greater overall satisfaction with the intervention. ${ }^{29}$ Similar results were observed in our sample, as $53.3 \%$ of the ACT group reported they loved the intervention compared to $25 \%$ of the education group. It should be acknowledged that both interventions lead to improvements in family life, and both interventions appear to have distinct advantages. ACT may produce greater patient satisfaction as it elicits more personal reflection. Additionally, ACT may be better suited for managing romantic relationships or losses in multiple life domains. Conversely, education may be considered a cost-effective intervention for those with fewer impacted life domains.

Regarding success in the work domain, both 
groups showed little change in success in the work domain over the duration of the study. More importantly, there was no meaningful change in reported success for either group. It is possible that the lack meaningful change in success in the work domain may be due to the high rates of disability and unemployment in the sample. Given that the majority of the sample was not working, success in work may not be relevant to these individuals.

The findings indicate that ACT may be quick to elicit change in success during the intervention; however, these improvements are not maintained after completion. Other ACT interventions with chronic pain populations have found maintained improvements in valuesbased action/success in values which were maintained. ${ }^{25,29}$ Notably, these studies had active intervention periods that were longer in total duration or more intensive as part of inpatient pain management programs. Thus, it may be that more intensive ACT results in better maintained effects.

\section{Future directions/clinical implica- tions}

The results of this small pilot study provide preliminary insight into ACT as intervention for FMS, which may lead to greater success in important life domains. However, the results of this study should be confirmed with a larger sample and replication is needed. Long-term follow up is needed to assess the trajectory of change for each intervention, as one may prove to have lasting effects and thus become an optimal choice of treatment. Additionally, attention should be given to the malleability of life domains; it may be that family relationships are somewhat easier to change in comparison to intimate relationships. If so, this would certainly play a role in the interpretation of the present findings; however this is beyond the scope of this paper.

\section{Limitations}

As with any small study, the ability to detect group differences was hindered by limited power. However, our noted effect sizes (Table 2) suggest power did not significantly impact our ability to test the hypotheses. This pilot was conducted with a fairly homogenous sample. As such, it is possible that results may not generalize to others with FMS. Statistics show that the demographics of our sample are comparable to those of other FMS studies. ${ }^{21}$ However, future research should examine our findings with a more demographically diverse set of patients. Related to this concern, selection bias may have impacted this sample. Given that the participants in this study were willing to travel to the medical center once a week; it is possible that those in the sample had less intense symptoms than the general
FMS population, creating greater willingness to travel.

\section{Conclusions}

These findings show that FMS patients are able to improve their success in family and intimate relationships. This is important as these relationships can be significantly hindered by FMS. The results of this study seem to indicate that both ACT and education on symptom self-management may be effective at improving success in family, and ACT may be especially appropriate for targeting success in intimate relationships. Both interventions may have distinct advantages (education is cost effective, and ACT promotes patient satisfaction), and may be differentially suited for different life domains. Although their lives may not be entirely free of pain or fatigue, people with FMS can learn to be more successful in life domains valuable to them, and this change can take place through multiple types of interventions.

\section{References}

1. Wolfe F, Ross K, Anderson J, et al. The prevalence and characteristics of fibromyalgia in the general population. Arthritis Rheum 1995;38:19-28.

2. Desmeules JA, Cedraschi C, Rapiti E, et al. Neurophysiologic evidence for central sensitization in patients with fibromyalgia. Arthritis Rheum 2003;48:1420-9.

3. Soderberg S, Lundman B. Transitions experienced by women with fibromyalgia. Health Care Women Int 2001;22:617-31.

4. Preece JC, Sandberg JG. Family resilience and the management of fibromyalgia: Implications for family therapists. Contemp Fam Ther 2006;27:559-76.

5. Romano JM, Turner JA, Jensen MP. The family environment in chronic pain patients: Comparison to controls and relationship to patient functioning. J Clin Psychol Med Settings 1997;4:383-95.

6. Arnold LM, Crofford LJ, Mease PJ, et al. Patient perspectives on the impact of fibromyalgia. Patient Educ Couns 2008;73: 114-20.

7. Soderberg S, Strand M, Haapaa M, Lundman B. Living with a woman with fibromyalgia from the perspective of the husband. J Adv Nurs 2003;42:143-50.

8. Steiner JL, Bigatti SM, Hernandez AM, et al. Social support mediates the relations between role strains and marital satisfaction in husbands of patients with fibromyalgia syndrome. Fam Syst Health 2010;28:209-23.

9. Oliver K, Cronan TA, Walen HR, Tomita M. Effects of social support and education on health care costs for patients with fibromyalgia. J Rheumatol 2001;28:2711-9.

10. Bigatti SM, Lydon JR, Brothers BM. Role strains and mood in husbands of women with fibromyalgia syndrome: a test of the stress process model. Open Fam Stud J 2008;1:66-75.

11. Henriksson, CM, Liedberg GM, Gerdle B. Women with fibromyalgia: work and rehabilitation. Disabil Rehabil 2005;27:685-95.

12. Shaver JLF, Wilbur J, Robinson FP, et al. Women's health issues with fibromyalgia syndrome. J Womens Health 2006;15:103545.

13. Burckhardt CS, Liedberg GM, Henriksson CM, Kendall S. The impact of fibromyalgia on employment status of newly-diagnosed young women: a pilot study. J Musculoskelet Pain 2005;13:31-41.

14. Kurtze N, Gundersen KT, Svebak S. The impact of perceived physical dysfunction, health-related habits, and affective symptoms on employment status among fibromyalgia support group members. J Musculoskelet Pain 2001;92:39-53.

15. Hayes SC, Strosahl K, Wilson KG. Acceptance and commitment therapy: an experiential approach to behavior change. New York: Guilford Press; 1999.

16. Hayes SC, Strosahl K, Wilson KG. Acceptance and commitment therapy, 2nd ed. The Process and Practice of Mindful Change. New York: Guilford Press; 2012.

17. Viane I, Crombez G, Eccleston C, et al. Acceptance of pain is an independent predictor of mental well-being in patients with chronic pain: empirical evidence and reappraisal. Pain 2003;106:65-72.

18. McCracken LM. Learning to live with the pain: acceptance of pain predicts adjustment in persons with chronic pain. Pain 1998;74:21-7.

19. McCracken LM, Vowles KE, Eccelston C. Acceptance-based treatment for persons with complex, long standing chronic pain: a preliminary analysis of treatment outcome in comparison to a waiting phase. Behav Res Ther 2005;43:1335-46.

20. Dahl J, Wilson KG, Nilsson A. Acceptance and commitment therapy and the treatment of persons at risk for long-term disability resulting from stress and pain symptoms: a preliminary randomized trial. Behav Ther 2004;35:785-801.

21. Ang DC, Kaleth AS, Bigatti S, et al. Research to Encourage Exercise for Fibromyalgia (REEF): use of motivational interviewing design and method. Contemp Clin Trials 2011;32:59-68.

22. McCracken LM, Yang $S$. The role of values 
in a contextual cognitive-behavioral approach to chronic pain. Pain 2006;123:137-45.

23. Garamszegi LZ. Comparing effect sizes across variables: generalization without the need for Bonferroni correction. Behav Ecol 2006;17:682-7.

24. Peterson BD, Eifert GH, Feingold T, Davidson S. Using acceptance and commitment therapy to treat distressed couples: a case study with two couples. Cogn Behav Pract 2009;16:430-42.

25. Vowles KE, McCracken LM. Acceptance and values-based action in chronic pain: A study of treatment effectiveness and process. J Consult Clin Psychol 2008;76:397-407.

26. Nicassio PM, Radojevic V, Weisman MH, et al. A comparison of behavioral and educational intervention for fibromyalgia. $\mathrm{J}$ Rheumatol 1997;24:2000-7.

27. Hassett AL, Gervitz RN. Nonpharmacologic treatment for fibromyalgia: patient education, cognitive behavioral therapy, relaxation techniques, and complementary medicine. Rhuem Dis Clin North Am
2009;35:393-407.

28. Turk DC, Okifiki A, Sinclair JD, Starz TW. Interdisciplinary treatment for fibromyalgia syndrome: clinical and statistical significance. Arthritis Rheum 2005;11:18695.

29. Wetherell JL, Afari N, Rutledge T, et al. A randomized, controlled trial of acceptance and commitment therapy and cognitivebehavioral therapy for chronic pain. Pain 2001;152:2098-107. 